\title{
Antecedentes para a Visitação de Eventos Culturais no Bairro do Recife
}

\section{Resumo}

\author{
Alessandra Souza Queiroz Meloa \\ Marconi Freitas da Costab
}

Essa investigação teve o propósito de mensurar qual influência os grupos de referência (GDR), a sensibilidade ao preço (SAP) e a identificação (I) poderiam exercer sobre a intenção de visitar eventos culturais em espaços públicos do Bairro do Recife. Como estratégia de pesquisa foi utilizado o survey online. Um questionário estruturado foi desenvolvido, obtendo 116 respondentes, todos considerados válidos para a amostra final. Os dados foram analisados com base na análise de regressão linear múltipla. Assim, os construtos de identificação (I) e grupos de referência (GDR) influenciam positivamente na decisão de visitar eventos culturais em espaços públicos, já o construto de sensibilidade à preço (SAP) não exerce influência alguma sobre a VD intenção de visitar eventos culturais em espaços públicos do Bairro do Recife. As características do público que frequenta o Bairro do Recife, não são influenciadas por aspectos financeiros, são influenciadas por grupos de referência e pela identificação com a localidade. A relevância desse trabalho se encontra em sua originalidade a temática.

Palavras-chave: Sensibilidade ao preço; Grupos de referência; Identificação; Eventos culturais.

\section{Abstract \\ Background to Visit Cultural Events in the Bairro do Recife}

This investigation aimed to measure which influence the reference groups (GDR), price sensitivity (SAP) and identification (I) could have on the intention to visit cultural events in public spaces in the Bairro do Recife. The online survey was used as a research strategy. A structured questionnaire was developed, obtaining 116 respondents, all considered valid for the final sample. The data were analyzed based on multiple linear regression analysis. Thus, the identification constructs (I) and reference groups (GDR) positively influence the decision to visit cultural events in public spaces, whereas the price sensitivity construct (SAP) has no influence on the RV intention to visit cultural events in public spaces in Bairro do Recife. The characteristics of the public that frequents Bairro do Recife are not influenced by financial aspects, they are influenced by reference groups and by identification with the locality. The relevance of this work is found in its originality the thematic.

Keywords: Price sensitivity; Reference groups; Identification; Cultural events; Urban public space.

a. Mestranda no Programa de Pós-graduação em Hotelaria e Turismo da Universidade Federal de Pernambuco. Recife, Pernambuco, Brasil. E-mail: alessandra_queirozz@outlook.com

b. Doutor em Administração pela Faculdade de Economia e Administração da Universidade de São Paulo. Docente do Programa de Pós-Graduação em Gestão, Inovação e Consumo - PPGIC na Universidade Federal de Pernambuco (UFPE). Caruaru, Pernambuco, Brasil. E-mail: marconi.focsta@ufpe.br

c. Doutor em Administração pela Universidade Federal de Pernambuco. Professor Adjunto do Curso de Administração e Programa de Pós-graduação em Hotelaria e Turismo da Universidade Federal de Pernambuco. Recife, Pernambuco, Brasil. E-mail: j.roberto.guerra@gmail.com 


\section{Resumen}

\section{Antecedentes para visitar eventos culturales en el Bairro do Recife}

Esta investigación tuvo como objetivo medir qué influencia podrían tener los grupos de referencia (GDR), la sensibilidad al precio (SAP) y la identificación (I) sobre la intención de visitar eventos culturales en espacios públicos en el Bairro do Recife. La encuesta en línea se utilizó como estrategia de investigación. Se elaboró un cuestionario estructurado, obteniendo 116 encuestados, todos considerados válidos para la muestra final. Los datos se analizaron en base a un análisis de regresión lineal múltiple. Así, los constructos de identificación (I) y los grupos de referencia (GDR) influyen positivamente en la decisión de visitar eventos culturales en espacios públicos, mientras que el constructo de sensibilidad al precio (SAP) no influye en la intención de RV de visitar eventos culturales. en espacios públicos en Bairro do Recife. Las características del público que frecuenta el Bairro do Recife no están influenciadas por aspectos económicos, están influenciadas por grupos de referencia y por identificación con la localidad. La relevancia de este trabajo se encuentra en su originalidad la temática.

Palabras clave: Sensibilidad al precio; Grupos de referencia; Identificación; Eventos culturales.

\section{INTRODUÇÃO}

Os eventos culturais em espaços públicos urbanos ao longo do tempo sofreram modificações, tanto no que diz respeito ao seu público, ao trazer movimento de pessoas de grupos sociais distintos a compartilharem o mesmo espaço, quanto à intensidade das realizações desses eventos. 0 que antes era algo esporádico, hoje é trabalhado como uma estratégia mercadológica para atrair pessoas (Sevcenko, 2002). É nesse cenário de mudanças, onde há vários tipos de pensamentos e formas de expressão, que os eventos culturais são produzidos (Paiva, 2016). A construção dos espaços públicos é um processo contínuo e marcado por conflitos e interesses, quanto mais as pessoas migram para as cidades, mais as cidades se tornam um cenário de disputa e conquista. Nesse processo, os avanços tecnológicos da comunicação e informação, marcam o início de novas formas de consumo, planejamento, vivência e locomoção, criando práticas de comunicação e representação que formam culturas urbanas distintas, construindo espaços mediados e midiatizados, representados por diferentes formas de consumo e utilização, a depender dos agentes que as movimentam (Castells \& Borja, 1996).

Diante do exposto, o Bairro do Recife localizado na cidade do Recife, capital Pernambucana, é um exemplo de disputas e conquistas por espaços urbanos. Ele é marcado por conflitos entre os interesses públicos, privados e dos moradores locais, que buscam garantir seus espaços no Bairro. Ao realizarem eventos culturais nos espaços públicos do Bairro do Recife, a forma de percepção da localidade é alterada, de acordo com Barroso e Fernandes (2018), localidades como essa, ao serem modificadas em suas dinâmicas urbanas, atribuindo em seus entornos eventos culturais, despertam na população a sensação de um local de vivência, encontros, ocupação e permanência.

Pearce (2001), destaca que a convivência entre atrações culturais, históricas, patrimônio arquitetônico, as experiências de entretenimento, passeios, gastronomia, etc., tornam as cidades destinos considerados completos, formando assim a cidade turística. A qual se apresenta como uma nova forma de urbanização, 
essas cidades não são concebidas para a produção, como por exemplo, são as cidades industriais. A cidade turística traz a ideia de ser produzida para o consumo de serviços, bens e paisagens. 0 autor ainda relata que esse turismo urbano tem suas raízes a partir dos fenômenos de urbanização, onde muitas pessoas se deslocavam para as cidades em busca de desfrutar as multiplicidades de coisas, desde arte, cultura, literatura, a arquiteturas e designs urbanos. Jansen-Verbeke (1988), compartilha desse pensamento bem antes das contribuições de Pearce, desse modo, a autora considera componentes do turismo urbano, os edificados históricos, as paisagens urbanas, assim como os espetáculos e eventos que ocorrem nesses lugares, chegando a afirmar que esses elementos, possivelmente são os motivadores das visitações dos turistas nas cidades.

Trazendo as contribuições de Leite e Peixoto (2009), compreendemos que a partir de 2001, segundo os autores, o Bairro do Recife teve o seu enobrecimento, onde suas feições arquitetônicas foram bastante modificadas, passando de casarões para animados e sofisticados restaurantes, suas ruas tornavam-se agora polco de espetáculos teatrais, exposições artísticas, shows, entre outros, tornando o do Bairro do Recife boulevards, ou seja, uma via urbana larga, projetada com algum objetivo paisagístico, para aquelas famílias de classe média da cidade. Assim, o bairro passou de um lugar marginalizado, para um lugar de lazer, segurança, encontros, entretenimento e palco de eventos culturais e políticos (Leite \& Peixoto, 2009). Atualmente, os eventos culturais acontecem com maior frequência no Bairro do Recife, atraindo diversos públicos. Esses eventos fazem com que ocorra interatividade entre as pessoas e os espaços públicos do bairro, estimulando o turismo urbano, uma vez que nesses espaços há um grande fluxo de pessoas de diferentes classes sociais e níveis de renda. Acredita-se que o perfil dos eventos, por serem gratuitos para todos os públicos, atraiam pessoas sensíveis à preço. Esta sensibilidade, de acordo com Lichtenstein, Ridgway e Netemeyer (1993), refere-se às expressões do consumidor em procurar, se necessário, por preços mais baixos de determinados produtos. Ou seja, se o consumidor de fato está disposto a tentar um esforço extra para conseguir preços mais baixos.

Outro fator que afeta a intenção de visitar um evento cultural são os grupos de referências, que são definidos por grupos de pessoas, ou indivíduos, que possuem relevância significativa nas avaliações ou comportamentos que afetam na decisão dos indivíduos. Os grupos de referências desenvolvem um papel importante ao servirem de modelo para os indivíduos que ajustam seus valores, crenças e comportamentos, as normas especificas de cada grupo, sua importância vai além, ao dar continuidade a socialização dos papéis que a humanidade desenvolve na vida em grupo (Zapata Muñoz, Puerta Campiño \& Restrepo, 2018).

Com relação ao fator de identificação, acredita-se que este exerce influência sobre o indivíduo na intenção de visitar um espaço (evento) público, uma vez que esse construto diz respeito à força de relacionamento que o indivíduo possui com a marca, nesse caso com o lugar. 0 quão aquele lugar possui particularidades semelhantes com as do indivíduo (Algesheimer, Dholakia \& Herrmann, 2005). A identificação desperta as semelhanças e particularidades que o indivíduo possui com o local. Muitas vezes, há um conjunto de fatores que são determinantes na identificação, sendo considerados não apenas as semelhanças que o indivíduo possui com o local, mas, com outros indivíduos ali presentes, com fatores históricos que despertam emoções e trazem sensações de pertença, etc. Dessa forma, 
a identificação diz respeito ao autoconceito que pode derivar do pertencimento à localidade ou aos indivíduos que a compõem e do valor emocional atribuído a ela (Nascimento et al., 2017).

A principal contribuição desta pesquisa consiste na proposição de possíveis antecedentes, tais como, sensibilidade à preço, grupos de referência e identificação, que supostamente explicam o comportamento dos indivíduos ao visitarem eventos culturais nos espaços públicos do Bairro do Recife. Estes construtos não foram abordados anteriormente em pesquisas na área acadêmica que contemplam esta temática e da forma analisada. Tal constatação justifica a realização desse estudo e busca contribuir para as discursões literárias a cerca desta temática.

Portanto, este trabalho contribui para a área acadêmica em avanços científicos, servindo como fonte para estudos futuros nas áreas de gestão pública, turismo e urbanismo. Do ponto de vista mercadológico o trabalho pode contribuir com dados atuais que colaboram com as estratégias do mercado de eventos culturais, contribuindo com dados e insights que ajudem na implementação adequada das estratégias para a gestão do turismo urbano e dos espaços públicos. Para o mercado tecnológico, esse estudo contribui com informações e dados que podem auxiliar as estratégias de implementação de novas tecnologias urbanas. Além disso, o trabalho academicamente contribui por ser uma temática pouco discutida com tal enfoque. Essa pesquisa foi realizada com a finalidade de identificar qual a influência que a sensibilidade à preço (SAP), grupos de referências (GDR), e a identificação (I), exercem sobre a intenção de visitar eventos culturais em espaços públicos urbanos do Bairro do Recife.

\section{Turismo Urbano e os eventos culturais no Bairro do Recife}

Henriques (2003), escreve sobre a complexidade e antiguidade da relação do turismo com o espaço urbano, afirmando que, por muitas vezes, em estudos científicos é omitido que o turismo em sua essência é um fenômeno urbano e pertence a urbanistas com seus vários graus urbanizados. Mas, não se pode negligenciar que o turismo é um acontecimento; que, de acordo Pearce (2011), à medida em que a população avança com seu modo de vida, pode ser observado com maior intensidade. Nesse contexto, os autores Henriques (2003) e Pearce (2011), sustentam a ideia de que a urbanização estimula o turismo ao mesmo tempo em que o turismo estimula a urbanização. Cada cidade terá o seu grau de atração, seja pelo patrimônio histórico, seja pela cultura, seja pela arquitetura; e tantas outras. Dessa forma, há um leque de oportunidades atrativas oferecidas pelas cidades, fazendo com que elas recebam públicos diversos, que as procuram por diferentes motivos (Henriques, 2003). Um desses motivos são os eventos culturais, que podem apresentar finalidades distintas, como, por exemplo, turismo, lazer, recreação, esportes e cinema (Getz, 2008). Os eventos culturais em espaços públicos urbanos criam uma dinâmica diferente da habitual aos locais, surgindo opções de lazer e recreação (Liu, 2014). Assim, fazem com que a cultura possa ser acessada por todas as classes sociais.

Os eventos realizados no Bairro do Recife representam em sua maioria um marco para a cultura Pernambucana, como é o caso do carnaval, onde estão concentrados no bairro os principais blocos carnavalescos do estado, os eventos 
tecnológicos também marcam o novo aspecto tecnológico atribuído ao bairro, promovendo os fluxos de pessoas e ambos tornam o bairro mais habitável. E vários outros eventos também promovem encontros e fluxos de pessoas no local.

Variados são os motivos pelos quais as pessoas frequentam eventos culturais do Bairro do Recife, segundo as contribuições de Getz e Page (2016), esses motivos podem ser relacionados aos estudos de Maslow ${ }^{1}$ sobre as necessidades humanas, compreendendo a necessidade em participar de eventos culturais em níveis diferentes, a começar por uma necessidade fisiológica, segundo por uma necessidade de segurança, terceiro por uma necessidade de amor ou relacionamento, quarto e quinto por uma necessidade de estima e realização pessoal, demonstrando assim, a importância dos eventos para o emocional e o afetivo dos indivíduos.

Os eventos também são importantes para fomentar o turismo e podem criar oportunidades de lazer para a comunidade local e regional, por trazerem diversas contribuições ao local, inclusive organizações privadas e governamentais tem interesse em reunir os impactos positivos ofertados por estes eventos (Macedo, Gosling \& Queiroz 2017). Nesse contexto, é provável que existam fatores que são influenciadores e determinantes para que esses eventos atraiam um número crescente de pessoas.

Nesse trabalho acredita-se que fatores como a sensibilidade ao preço, possam contribuir significativamente na decisão de visitar eventos culturais em espaços públicos, por possuir preços mais baixos ou simplesmente não exigir um investimento, sendo gratuitos (Trindade \& Souza, 2018). Outro fator que possivelmente contribui nessa tomada de decisão são os grupos de referência, onde o indivíduo será influenciado por esses grupos que podem ser formados por pessoas próximas ou por artistas e possuem um poder decisório sobre o indivíduo (Bragaglia \& Bastos, 2017).

0 fator de identificação, apresenta também alto potencial de influenciador para a tomada de decisões dos indivíduos. 0 indivíduo fará sua escolha de acordo com o quão ele se identifica com o local (Nascimento et al., 2017). A seguir serão apresentados cada um desses fatores, melhor dizendo construtos que supostamente exercem influência na intenção de visitar eventos culturais em espaços públicos.

\section{Sensibilidade à preço}

De acordo com Lichtenstein et al. (1993) a escala de sensibilidade ao preço diz respeito ao quanto as pessoas estão dispostas a procurar por produtos econômicos e se de fato elas fazem algum esforço para procurar esses produtos financeiramente baratos. 0 preço possui influência relevante quando o assunto é comportamento de compra do consumidor, por isso a promoção ou procura por preços mais baixos é um dos aspectos que vem crescendo na hora dos consumidores fazerem suas escolhas (Han, Gupta \& Lehmann, 2001). Em uma empresa, por exemplo, é necessário que os seus gestores estejam atentos a precificação

1. O psicólogo americano Abraham H. Maslow define cinco categorias de necessidades humanas, onde são representadas por uma pirâmide, que em sua base pode ser encontrada as necessidades básicas que estão ligadas a sobrevivência. As cinco categorias das necessidades humanas apresentadas por Maslow são: fisiológicas, segurança, amor/relacionamento, estima e realização pessoal. 
dos seus produtos, primeiro porque ajuda a gestão a escolher devidamente o preço que será estabelecido para seu produto, sem impactar os consumidores, segundo porque fornece aos gestores um método de segmentação dos consumidores (Trindade \& Souza, 2018).

Dessa forma, voltando tais efeitos para os eventos culturais em espaços públicos, pode-se dizer que a aplicabilidade da precificação nesses locais pode influenciar assim como nas empresas, tanto no impacto positivo quanto no impacto negativo, assim como no segmento dos consumidores. (Han et al., 2001). A sensibilidade à preço (SAP) influencia positivamente a intenção de visitar eventos culturais nos espaços públicos do Bairro do Recife, segundo os autores Han et al., (2001) há preços de referência que são parte da atividade da precificação, eles ficam armazenados na mente do consumidor, servindo de comparação a compras futuras, por exemplo, quando um consumidor efetua uma compra de algum produto e logo mais ele o encontra mais barato a sensação que ele irá ter é de perda, porém se o mesmo encontra o produto mais caro a sensação será de ganho. Com base no exposto, essa pesquisa formulou a seguinte hipótese:

$H_{1}$ : A sensibilidade à preço (SAP) influencia positivamente a intenção de visitar eventos culturais nos espaços públicos do Bairro do Recife.

\section{Grupos de referência}

Os grupos de referência assumem um papel de influenciador, considerando os fatores sociais, culturais, pessoais e psicológicos, é possível perceber que os indivíduos, buscam informações em fontes que já utilizam determinadas marcas ou que já consumiram determinados produtos, para realizar suas decisões finais de compra ou avaliar produtos. Desse modo, os indivíduos utilizam produtos e marcas como forma de transparecer a sua identidade e uma boa imagem, para que dessa forma possa garantir a aprovação social e a sensação de pertencimento a determinado grupo (Inglis \& Solomon, 1995).

Bragaglia \& Bastos (2017) colocam o consumo como o centro da sociedade moderna. Ao adquirir bens ou marca o indivíduo assume um relacionamento e posicionamento com as pessoas, ou grupos de pessoas que também usam as mesmas coisas. Os comportamentos e valores dos indivíduos são condicionados pelos respectivos grupos de referência (amigos, familiares, influenciadores digitais e artistas). Esses grupos despertam a sensação de pertencimento e reconhecimento, influenciando diretamente ou indiretamente os indivíduos. Normalmente, esses grupos fazem parte do nosso dia a dia, seja no trabalho, na faculdade, nas redes sociais ou em casa (Abreu, 2020).

Dessa forma, pode ser compreendido como grupo de referência, qualquer pessoa ou grupo de pessoas que assume o papel de comparação para um indivíduo na formação dos seus valores, atitudes e decisões (Escalas \& Bettman, 2003). Assim, os indivíduos são influenciados por diversos fatores que vão desde os individuais, psicológicos e ambientais (Inglis \& Solomon, 1995). Os seres humanos em sua essência são sociais, pois vivem e interagem diariamente, desse modo, as relações estabelecidas pautadas em influências interpessoais, interferem no comportamento e atitude dos indivíduos, em geral (Sastre, Serralvo \& Moras, 2010). 
Há grupos de referência que são classificados de diversas formas, e um indivíduo pode estar em mais de uma classificação, simultaneamente (Engel, Blackwell, \& Miniardi, 2005), classificam os grupos de referência os dividindo em: grupos primários e secundários, os grupos aspiracionais e dissociativos, grupos formais e informais, e os grupos virtuais. Um bom exemplo dos grupos primários é a família, uma vez que, esse grupo exerce maior influência e impacto na decisão de escolha dos indivíduos, isso acontece devido a frequência diária de interação estabelecida por esses grupos, que pode ocorrer frente a frente ou não.

Já os grupos secundários são compostos por parentes, sindicatos ou associações, e estabelecem uma interação menos frequente, suas normas e opiniões possuem menos impactos que as do grupo primário. Os grupos formais possuem um grau de abrangência e influência, a partir do valor que os indivíduos atribuem as opiniões do grupo. Um exemplo desse grupo são os religiosos e organizações comunitárias. Os grupos informais são formados pelos grupos de voluntários, amigos e pessoas que possuem os interesses em comum, esse tipo de grupo é baseado nos interesses em comum e na amizade (Sastre, Serralvo \& Moras, 2010). Os grupos aspiracionais são aqueles que estabelecem o desejo de adotar, condutas, normas e comportamentos de outros indivíduos que ambicionam se associar ou serem parecidos. Já os grupos dissociativos refletem o oposto dos grupos aspiracionais. Esses grupos evitam o contato e a associação. E os grupos virtuais, são aqueles que os indivíduos mantêm contato e interagem por meio virtual, um exemplo esses grupos nos dias atuais são as redes de relacionamentos Instagram e Facebook (Engel, Blackwell, \& Miniardi, 2005).

Desse modo, as avaliações e opiniões dos indivíduos ou grupos de indivíduos, de algum modo, inspiram o comportamento de outros, chegando à conclusão de que a opinião de terceiros é um dos grandes fatores do comportamento humano Escalas \& Bettman, 2003). De acordo com as pesquisas literárias realizadas para compor este estudo, verificou-se uma ausência em discursos a respeito da influência de escolha em eventos culturais estabelecidas pelos grupos de referência. Assim, buscou-se em estudos que não tratam especificadamente sobre a influência em ventos cultuais, mas sobre a influência desses grupos de modo geral na sociedade, trazendo para esta discussão o aporte teórico necessário. Essa ausência de aporte teórico sobre tal discussão, reforça ainda mais a importância desse estudo para as contribuições literárias. Diante do exposto, esta pesquisa formulou a segunda hipótese:

$H_{2}$ : Os grupos de referência (GDR) influenciam positivamente na intenção de visitar eventos culturais nos espaços públicos do Bairro do Recife.

\section{Identificação}

Para Algesheimer et al. (2005), a identificação diz respeito ao quão o indivíduo encontra na localidade particularidades semelhantes às dele. Dessa forma, a identificação encontra-se atrelada às necessidades, objetivos, conhecimentos, produto e valores do indivíduo. Quando há o reconhecimento de um produto ou lugar considerado de relevância pelo indivíduo, automaticamente é ativado na sua memória, criando um estado emocional que conduz a determinados 
comportamentos. Desse modo, a identificação do consumidor com o lugar ou produto o leva a procurá-los e consumi-los, ao contrário do baixo envolvimento com o local ou produto, onde faz com que o consumidor não apresenta disposição para informes sobre eles (Fonseca \& Rossi 1998).

Quando falamos sobre identificação, podemos refletir sobre a sociedade e os modos de vida que fazem parte dela, mesmo cada indivíduo sendo único em sua existência, somos parte integrante de um sistema maior, onde nos identificamos muitas vezes com os mesmos objetos ou lugares que outros indivíduos se identificam também, isso porque, a vida é feita de relações, logo é natural que o indivíduo ao longo de sua vida acabe se identificando com, por exemplo, aspectos arquitetônicos de um lugar, ou com as pessoas que frequentam um bairro, ou festividades (Nascimento, et al., 2017). De acordo com o mesmo autor, o ser humano, necessita da identificação para desenvolver o sentimento de pertencimento, dessa forma ele se sente inserido no meio social, e fará suas escolhas com base do quanto ele se encontra envolvido ou do quanto ele possui afinidade com o lugar.

A identificação pode ser um fator essencial para a tomada de decisão dos indivíduos. Nesse processo há estímulos que envolve o psicológico, estimulando aspectos que influenciam o emocional, assim, há várias formas de manifestar o sentimento de identificação com o local e cada indivíduo em sua particularidade irá ativar de maneira distinta (Algesheimer et al., 2005). Dessa forma, a pesquisa formulou a terceira hipótese:

$H_{3}:$ a identificação influencia positivamente na intenção de visitar eventos culturais nos espaços públicos do Bairro do Recife.

\section{Modelo teórico}

Este tópico visa apresentar sucintamente as relações discutidas e apresentadas anteriormente em cada hipótese. Assim, é possível visualizar como às três variáveis, sensibilidade ao preço, grupos de referência e identificação, supostamente influenciam os indivíduos na intenção de consumir eventos culturais em espaços públicos urbanos.

Figura 1 - Modelo teórico da investigação. H1: Hipótese 1; H2: Hipótese 2; H3: Hipótese 3



Fonte - Elaborado pelos autores (2019) 


\section{MÉTODO DA PESQUISA}

\section{População e amostra}

A população do estudo foi composta por pessoas brasileiras que já frequentou algum tipo de evento cultural no Bairro do Recife-PE, tendo como caráter a amostragem não probabilística, devido à falta de um quadro que demonstrasse todas as pessoas que já participaram. Foi utilizada a amostragem por conveniência, que se utiliza de indivíduos ou grupos de indivíduos que estejam disponíveis, esse tipo de amostra pode proporcionar informações preciosas da amostra assim construída (Carmo \& Ferreira, 2008).

Assim os participantes foram selecionados por critério pré-estabelecido: (a) ter participado de algum evento no Bairro do Recife. Para intensificar o número de respondentes, foi utilizado a técnica snowball, onde respondentes passavam o questionário para colegas e amigos, de modo a ampliar a amostra final. As pessoas foram acessadas pelas redes sociais, como Instagram, Facebook e pelo WhatsApp, após esse contato os respondentes foram solicitados para repassarem o questionário para os seus colegas. Pesquisas com amostra e população similares a este estudo podem ser utilizadas em diversas áreas do conhecimento para diferentes temáticas, como é o caso dos estudos de Horta, et al. (2016) e Souza, Guimarães e Araújo (2013), que trazem estudos similares.

\section{Procedimentos de coleta dos dados}

Para contemplar as necessidades do método descritivo survey online, foi utilizado como instrumento de coleta de dados o questionário estruturado, que teve o seu desenvolvimento a partir da plataforma Google Forms. Com base no referencial teórico mensurou-se o construto, sensibilidade ao preço de acordo com a escala desenvolvida por Lichtenstein et al. (1993), com cinco itens. 0 construto grupo de referência, com nove itens, também foi mensurado de acordo com a escala de Park \& Lessig (1977). Com relação ao construto, identificação com três itens, optou-se pela utilização da escala de Algesheimer et al. (2005). E, por fim, com relação intenção de visitar eventos culturais em espaços públicos, a variável dependente deste estudo, teve sua mensuração através da elaboração de três itens, desenvolvido pelos autores. Foi utilizada a base teórica de Putrevu \& Lord (1994) e Marciel, Maffezzolli e Martins (2018), para garantir a validade do construto.

As variáveis interdependentes e dependente tiveram sua mensuração a partir da escala de Likert com pontuações que variam entre 1 (concordo totalmente) a 7 (discordo totalmente). Foi desenvolvido uma tabela 1 para demonstrar os itens que fizeram parte do questionário, demonstrando assim cada construto e sua autoria. O questionário contou com 14 itens, distribuídos entre as variáveis independentes e a variável dependente e o perfil sociodemográfico. A Tabela 1 é formada pelos construtos, fonte de escala, itens (dimensões e variáveis) e os códigos que representam o construto. 0 código SAP, significa 'sensibilidade à preço', o código GDR, significa 'grupo de referência', o código I, faz relação ao construto de 'identificação' e o código IV significa 'intenção de visitar' Eventos Culturais em Espaços Públicos. A tabela também apresenta as fontes de escala, identificando os autores que foram utilizados para cada escala. Os itens da tabela correspondem as dimensões 
e variáveis de cada escala e são apresentados cada um. 0 código é uma forma de identificação de cada construto, serve para a sua identificação no corpo do texto.

Tabela 1 - Compreendendo o instrumento de coleta

\begin{tabular}{|c|c|c|c|}
\hline Construto & Fonte de escala & Itens (dimensões e variáveis) & Código \\
\hline \multirow[t]{5}{*}{$\begin{array}{l}\text { Sensibilidade } \\
\text { ao preço } \\
\text { (SAP) }\end{array}$} & \multirow[t]{5}{*}{$\begin{array}{l}\text { Lichtenstein } \\
\text { et al. (1993) }\end{array}$} & $\begin{array}{l}\text { Dimensão: consciência de preço } \\
\text { - Eu não estou disposto a fazer um esforço extra } \\
\text { para encontrar preços mais baixos }\end{array}$ & SAP01 \\
\hline & & $\begin{array}{l}\text { - Eu visito mais de um evento cultural público } \\
\text { para aproveitar os preços baixos }\end{array}$ & SAP02 \\
\hline & & $\begin{array}{l}\text { - O dinheiro economizado ao encontrar preços } \\
\text { mais baixos em eventos culturais em espaços } \\
\text { públicos geralmente não vale o tempo e o esforço* }\end{array}$ & SAP03 \\
\hline & & $\begin{array}{l}\text { - Eu nunca pesquisaria mais de um evento } \\
\text { cultural para encontrar preços baixos }\end{array}$ & SAP04 \\
\hline & & $\begin{array}{l}\text { - } 0 \text { tempo em que levo para encontrar preços } \\
\text { baixos geralmente não vale o esforço* }\end{array}$ & SAP05 \\
\hline \multirow[t]{9}{*}{$\begin{array}{l}\text { Grupo de } \\
\text { referência } \\
\text { (GDR) }\end{array}$} & \multirow[t]{9}{*}{$\begin{array}{l}\text { Park \& Lessig } \\
\text { (1977) }\end{array}$} & $\begin{array}{l}\text { Dimensão: Influência informacional } \\
\text { - Eu busco informações sobre vários eventos } \\
\text { culturais em espaços públicos com profissionais } \\
\text { ou grupos independentes de especialistas }\end{array}$ & GDR01 \\
\hline & & $\begin{array}{l}\text { - Eu busco informações daqueles que } \\
\text { profissionalmente trabalham com os } \\
\text { eventos culturais }\end{array}$ & GDR02 \\
\hline & & $\begin{array}{l}\text { - Eu busco conhecimento e experiência relacionado } \\
\text { à eventos culturais (comparando eventos culturais } \\
\text { A e eventos culturais B) daqueles amigos, vizinhos, } \\
\text { parentes ou colegas de trabalho que possuem } \\
\text { informações cofiáveis sobre o evento. }\end{array}$ & GDR03 \\
\hline & & $\begin{array}{l}\text { - O evento cultural que seleciono é influenciado pela } \\
\text { observação de testes que aprovem esses eventos } \\
\text { independentes de qual empresa faça o teste. }\end{array}$ & GDR04 \\
\hline & & $\begin{array}{l}\text { - A minha observação sobre os especialistas em } \\
\text { eventos culturais, influência na minha escolha. }\end{array}$ & GDR05 \\
\hline & & $\begin{array}{l}\text { - Para satisfazer as expectativas de colegas de } \\
\text { trabalho a minha decisão de visitar eventos } \\
\text { culturais em espaços públicos é influenciada por } \\
\text { suas referências }\end{array}$ & GDR06 \\
\hline & & $\begin{array}{l}\text { - A minha decisão de visitar eventos culturais em } \\
\text { espaços públicos é influenciada pelas referências } \\
\text { das pessoas com quem tenho interação social }\end{array}$ & GDR07 \\
\hline & & $\begin{array}{l}\text { - A minha decisão de visitar eventos culturais em } \\
\text { espaços públicos é influenciada pelas referências } \\
\text { dos membros da minha família }\end{array}$ & GDR08 \\
\hline & & $\begin{array}{l}\text { - O meu desejo de satisfazer as expectativas que } \\
\text { os outros possuem de mim, tem um impacto na } \\
\text { minha escolha por visitar eventos culturais em } \\
\text { espaços públicos }\end{array}$ & GDR09 \\
\hline
\end{tabular}


Tabela 1 - Continuação

\begin{tabular}{|c|c|c|c|}
\hline Construto & Fonte de escala & Itens (dimensões e variáveis) & Código \\
\hline \multirow[t]{3}{*}{$\begin{array}{l}\text { Identificação } \\
\text { (I) }\end{array}$} & \multirow{3}{*}{$\begin{array}{l}\text { Algesheimer; } \\
\text { Dholakia \& } \\
\text { Herrmann } \\
\text { (2005) }\end{array}$} & $\begin{array}{l}\text { - Os eventos culturais que frequento diz muito } \\
\text { sobre o tipo de pessoa que eu sou }\end{array}$ & I01 \\
\hline & & $\begin{array}{l}\text { - A imagem dos eventos culturais que frequento } \\
\text { e minha autoimagem são semelhantes em } \\
\text { muitos aspectos }\end{array}$ & I02 \\
\hline & & $\begin{array}{l}\text { - Os eventos culturais em espaços públicos } \\
\text { desempenham um papel importante na } \\
\text { minha vida }\end{array}$ & I03 \\
\hline \multirow{3}{*}{$\begin{array}{l}\text { Intensão } \\
\text { de visitar } \\
\text { eventos } \\
\text { culturais } \\
\text { em espaços } \\
\text { públicos (IV) }\end{array}$} & \multirow{3}{*}{$\begin{array}{l}\text { Putrevu \& Lord } \\
\text { (1994) adaptado } \\
\text { por Marciel, } \\
\text { Francisco- } \\
\text { Maffezzonlli, \& } \\
\text { Martins (2018) }\end{array}$} & $\begin{array}{l}\text { - Definitivamente vou visitar eventos culturais } \\
\text { em espaços públicos }\end{array}$ & IV01 \\
\hline & & $\begin{array}{l}\text { - É muito provável que eu visite eventos culturais } \\
\text { em espaços públicos }\end{array}$ & IV02 \\
\hline & & $\begin{array}{l}\text { - Vou visitar um evento cultural na minha } \\
\text { próxima viagem. }\end{array}$ & IV03 \\
\hline
\end{tabular}

Vale destacar que foi utilizado um pré-teste com 10 pessoas, com intuito de aprimoramento do instrumento de coleta, onde as contribuições dos participantes foram importantes nesse processo. Entretanto, não foi necessário fazer alterações no pré-teste, todos os participantes compreenderam cada processo e não tiveram dificuldades em responder. Isso demostra que na parte do pré-teste o estudo já estava refinado, não havendo a necessidade de alterações. Logo após esse processo de aperfeiçoamento, foi disponibilizado o link de acesso do questionário nas redes sociais (Facebook e WhatsApp).

\section{APRESENTAÇÃo e discussão dos Resultados}

De modo a identificar possíveis falhas dessa pesquisa, foram revisados os questionários resultantes, descartando o erro de não resposta (Porter \& Whitcomb, 2005) devido à obrigatoriedade de contemplar todos os itens do questionário, através do seu preenchimento na plataforma Google Forms. Dessa forma, foram realizados a confiabilidade e dimensionalidade das variáveis de todos os questionários respondidos $(n=116)$ e considerados todos os itens em sua totalidade. Em um segundo momento foram importados os dados para o software SPSS, seguindo com a análise através do software.

\section{Perfil sociodemográfico dos respondentes}

No início da análise com o propósito de obter um resumo simples sobre a amostra e as observações que foram realizadas, contabilizou-se o perfil sociodemográfico dos respondentes, utilizando-se de estatística descritiva simples, uma vez que essa estatística conta com a aplicação de várias técnicas para 
sumarizar conjuntos de dados da população (Reis \& Reis, 2002). Para demonstrar os dados com facilidade e determinar a ordem de importância dos valores que foram encontrados, foi obtido tais resultados baseados em frequência. Os resultados apresentados na tabela 2, demonstram que 50,9\% dos respondentes dessa pesquisa são do sexo feminino sobressaindo a porcentagem de 49,1\% referente ao sexo masculino.

Tabela 2 - Perfil sociodemográfico baseado em frequência

\begin{tabular}{|l|c|}
\hline \multicolumn{2}{|c|}{ Nível de escolaridade } \\
\hline Ensino médio & 26,7 \\
\hline Ensino superior & 34,5 \\
\hline Pós-graduação Lato Sensu (especialização, MBA) & 22,4 \\
\hline Pós-graduação Stricto Sensu (Mestrado, Doutorado) & 16,4 \\
\hline \multicolumn{1}{|c|}{ Sexo } & 50,9 \\
\hline Feminino & 49,1 \\
\hline Masculino & \\
\hline
\end{tabular}

Fonte - Pesquisa de campo (2019).

Ainda na Tabela 2, pode ser observado com relação ao nível de escolaridade dos respondentes desta pesquisa $34,5 \%$ possuem ensino superior, diferindo dos demais resultados onde 26,7\% possuem ensino médio, 22,4\% Pós-graduação Lato Sensu (especialização, MBA) e 16,4\% Pós-graduação Stricto Sensu (Mestrado, Doutorado). Sobre o perfil sociodemográfico dos respondentes, algumas das variáveis foram extraídas em escala razão para melhor comparação dos resultados, obtendo assim, maior precisão numérica a partir da média e do desvio padrão. Na Tabela 3, estão sintetizados os informes.

Tabela 3 - Perfil sociodemográfico baseado em médias e desvio padrão

\begin{tabular}{|l|c|c|}
\hline \multicolumn{1}{|c|}{ Variáveis } & Média & Desvio padrão \\
\hline Renda mensal (individual) & $\mathrm{R} \$ 8.257,43$ & $\mathrm{R} \$ 35.537,24$ \\
\hline Idade & 31,18 & 10,016 \\
\hline $\begin{array}{l}\text { Quantas vezes nesse ano você participou de eventos } \\
\text { culturais no Bairro do Recife }\end{array}$ & 3,59 & 3,749 \\
\hline
\end{tabular}

Fonte - Pesquisa de campo (2019).

A variável correspondente a renda mensal por indivíduo é de $\mathrm{R} \$ 8.257,43$ mil, um valor muito acima da renda média do estado que é $\mathrm{R} \$ 1,5$ mil, o que implica dizer que, de alguma forma a coleta de dados pode ter direcionado para esse público, uma vez que, a coleta foi realizada através do processo de Snowball (bola de neve), essa forma de coleta, pode ter trazido algum viés na amostra, para um público com renda mais elevada, um desses possíveis públicos são os colaboradores das empresas pertencentes ao parque tecnológico, Porto Digital, que se encontra instalado em todo o Bairro do Recife. A variável referente a média de idade é de 31,18 anos. Também foi identificado a variável que resulta em quantas vezes nesse ano (2019) os indivíduos participaram de algum evento cultural no Bairro 
do Recife, obtendo como resultado a média de 3,59 vezes. Após ser traçado o perfil sociodemográfico os esforços estatísticos agora serão direcionados para a consciência dos itens do questionário. Assim, na sessão seguinte serão apresentados resultados que são pré-requisitos para a realização da análise de regressão múltipla, descrevendo assim, todo o processo que foi realizado.

\section{Confiabilidade dos itens e dimensionalidade dos dados}

Com finalidade de verificar a confiabilidade dos itens, foi mensurado o Alfa de Cronbach, "medida de confiabilidade que varia de 0 a 1 , sendo os valores de 0,60 a 0,70 considerados o limite inferior de aceitabilidade" (Hair et al., 2009). Dessa forma, foi analisado o alfa em cada construto que consiste no modelo teórico da pesquisa, obtendo-se, assim, níveis considerados aceitáveis a partir de 0,60 . Nesse momento da análise apenas o construto identificação obteve um valor abaixo do aceitável 0,565, porém, devido a sua relevância teórica o construto foi mantido no modelo, mas os dados devem ser considerados com parcimônia, ou seja, porque os dados não podem ser levados em consideração como uma realidade absoluta, uma vez que, alguns índices não deram de acordo com o previsto, com o pressuposto da estatística, e também por causa até do próprio viés que tem na amostra.

Na sequência, para garantir que os indicadores propostos pela escala se encontram alinhados com a dimensão dos construtos foi realizada a análise fatorial; foram realizados os testes de Kaiser-Meyer-Olkin (KMO) que é um teste no qual sugere proporções de variância dos itens que possam estar sendo explicados por alguma variável latente. Na regra para interpretar os dados do KMO, valores menores que 0,5 são considerados inaceitáveis, entre 0,5 e 0,7 são considerados pobres, entre 0,7 e 0,8 são considerados bons, e os valores maiores que 0,8 e 0,9 são considerados ótimos (Pasquali, 1998; Hutcheson \& Sofroniou, 1999). Foi realizado também o teste de esfericidade de Bartlet (Dziuban \& Shirkey, 1974) que avalia se a medida da matriz de variância é parecida com a matriz-identidade, não apresentando correlação entre si. 0 teste de esfericidade é considerado adequado quando o Sig é menor que 0,05. Para facilitar a compreensão do que foi exposto, os dados foram sintetizados como mostra a Tabela 4.

Tabela 4 - Resumo da confiabilidade e dimensionalidade dos dados

\begin{tabular}{|c|c|c|c|c|c|c|c|c|}
\hline \multirow{2}{*}{ Variáveis } & \multirow{2}{*}{ C.F. } & \multirow{2}{*}{$\mathbf{H}^{2}$} & \multirow{2}{*}{ Кмо } & \multicolumn{3}{|c|}{ Bartlet } & \multirow{2}{*}{ V.Exp.* } & \multirow{2}{*}{ Cronbach } \\
\hline & & & & df & Qui $^{2}$ & Sig & & \\
\hline $\begin{array}{l}\text { IV01 } \\
\text { IV02 } \\
\text { IV03 }\end{array}$ & $\begin{array}{l}0,924 \\
0,926 \\
0,812\end{array}$ & $\begin{array}{l}0,854 \\
0,858 \\
0,660\end{array}$ & 0,683 & 3 & 197,111 & 0,000 & 79,057 & 0,865 \\
\hline $\begin{array}{l}\text { SAP01 } \\
\text { SAP02 } \\
\text { SAP03 } \\
\text { SAP04 } \\
\text { SAP05 }\end{array}$ & $\begin{array}{l}0,864 \\
0,851 \\
0,808 \\
0,623 \\
0,986\end{array}$ & $\begin{array}{l}0,423 \\
0,973 \\
0,731 \\
0,654 \\
0,748\end{array}$ & 0,767 & 10 & 154,224 & 0,000 & 50,295 & 0,675 \\
\hline
\end{tabular}


Tabela 4 - Continuação

\begin{tabular}{|c|c|c|c|c|c|c|c|c|}
\hline \multirow{2}{*}{ Variáveis } & \multirow{2}{*}{ C.F. } & \multirow{2}{*}{$\mathbf{H}^{2}$} & \multirow{2}{*}{ KMO } & \multicolumn{3}{|c|}{ Bartlet } & \multirow{2}{*}{ V.Exp.* } & \multirow{2}{*}{ Cronbach } \\
\hline & & & & df & Qui $^{2}$ & Sig & & \\
\hline $\begin{array}{l}\text { GDR01 } \\
\text { GDR02 } \\
\text { GDR03 } \\
\text { GDR04 } \\
\text { GDR05 } \\
\text { GDR06 } \\
\text { GDR07 } \\
\text { GDR08 } \\
\text { GDR } 09\end{array}$ & $\begin{array}{l}0,675 \\
0,719 \\
0,695 \\
0,673 \\
0,660 \\
0,657 \\
0,567 \\
0,508 \\
0,576\end{array}$ & $\begin{array}{l}0,700 \\
0,797 \\
0,754 \\
0,554 \\
0,605 \\
0,757 \\
0,826 \\
0,748 \\
0,804\end{array}$ & 0,768 & 36 & 421,203 & 0,000 & 41,849 & 0,822 \\
\hline $\begin{array}{l}\text { I01 } \\
\text { I02 } \\
\text { I03 }\end{array}$ & $\begin{array}{l}0,880 \\
0,870 \\
0,605\end{array}$ & $\begin{array}{l}0,757 \\
0,774 \\
0,367\end{array}$ & 0,587 & 3 & 84,935 & 0,000 & 63,254 & ,565 \\
\hline
\end{tabular}

* V.Exp. = variância explicada; $\mathrm{KMO}=$ Kaiser Meyer Olkin; $\mathrm{H}^{2}=$ comunalidade; $\mathrm{CF}=$ coeficiente; $\mathrm{DF}=$ graus de liberdade; $\mathrm{Qui}^{2}=$ qui-quadrado; Sig = Nível de significância ou P-Valor

Fonte - Pesquisa de campo (2019).

O primeiro construto a ser mensurado foi a intenção de compra, esse construto apresenta apenas uma única dimensão. Após o teste de confiabilidade dos itens do alfa de Cronbach que resultou em 0,867, sem a necessidade de retirada de itens, o índice de KMO foi extraído, demonstrando a validação com o valor de 0,683. 0 teste de Bartlet apresentou o qui-quadrado de 197,111 tendo 3 graus de liberdade e 0,000 de significância, também foi identificado que as cargas fatoriais desse construto indicaram que, em geral, todos os itens carregaram em um único fator e com cargas acima de 0,5. 0 teste de esfericidade de Bartlett busca avaliar a hipótese de as variáveis não serem correlacionadas na população, por meio da verificação da igualdade da matriz de identidade. Um valor do Sig, igual ou menor a 0,05 é considerado aceitável porque mostra a existência de correlação para algumas variáveis (Fávero et al., 2009).

0 segundo construto analisado foi a sensibilidade ao preço, tendo uma única dimensão o construto obteve, após o teste de confiabilidade dos itens do alfa de Cronbach de 0,675, índice KMO de 0,767, assim, não teve a necessidade de retirada de itens. 0 teste de Bartlet apresentou um qui-quadrado de 154,224, foi analisado que as cargas fatoriais desse construto carregaram em um único fator, obtendo 0,000 de significância e 10 graus de liberdade.

0 terceiro construto analisado foi o de grupos de referências, tendo uma única dimensão, o Alfa de Cronbach teve como resultado o valor de 822, sem a necessidade de se retirar itens, foi verificado o valor de 421,203 referente ao qui-quadrado do teste de Bartlet, as cargas fatoriais do construto GDR carregaram em um único fator. Obtendo um grau de liberdade de 36 e 0,000 de significância.

0 quarto e último construto a ser identificado foi identificação, esse construto apresenta uma única dimensão, e resulta nos valores referentes ao alfa de Cronbach de 0,565, sem a necessidade de retirar itens. Dessa forma, o teste de Bartlet foi realizado e obteve como resultado 84,935 referente ao qui-quadrado, com 3 graus de liberdade e 0,000 de significância. 


\section{Análise do modelo teórico proposto}

Com os itens que compuseram os construtos desse trabalho já tratados, eles foram agrupados como variáveis compostas (summated scale), para que dessa forma fosse realizado o processo de regressão, onde se define as relações das variáveis independentes com a dependente (Costa, Costa \& Silva, 2016). De acordo com a tabela 5 , a média de respostas dos construtos foi considerada intermediária, considerando a escala aplicada de Likert de 1 (discordo totalmente) e 7 (concordo totalmente). Notando que apenas a intenção de visitar e a identificação obteve uma pontuação alta, indicando, assim, um alto nível de concordância dos respondentes do questionário com as afirmativas desses construtos. Desse modo, é possível perceber com base na média da intenção de visitar, que as pessoas possuem um elevado interesse em visitar o Bairro do recife com relação a Sensibilidade ao preço que obteve uma média de 3,22 demostrando que essas pessoas não são sensíveis ao preço. 0 grupo de referência obteve a média 4,05 demostrando que as pessoas visitam o bairro por influência de outras pessoas ou grupo de pessoas. Já a identificação corresponde a uma média de 4,42 demostrando que essas pessoas frequentam o Bairro do Recife porque se identificam com o lugar.

Tabela 5 - Estatística descritiva das variáveis compostas

\begin{tabular}{|l|c|c|c|}
\hline \multicolumn{1}{|c|}{ Variáveis } & N & Média & D.P.* \\
\hline Intenção de visitar & 116 & 5,21 & 1,622 \\
\hline Sensibilidade ao preço & 116 & 3,22 & 1,287 \\
\hline Grupo de referência & 116 & 4,05 & 1,226 \\
\hline Identificação & 116 & 4,42 & 1,890 \\
\hline
\end{tabular}

*D.P. = desvio-padrão; $\mathrm{N}$ = número de respondentes.

Fonte - Pesquisa de campo (2019).

Utilizando o método Backward, que incorpora inicialmente todas as variáveis e depois, por etapas, cada uma pode ser eliminada, foi possível chegar aos resultados que demonstram, de acordo com a Tabela 6, a retirada da variável sensibilidade à preço, chegando à conclusão que o construto SAP não exerce influência considerada estatisticamente significativa com o modelo teórico proposto neste trabalho, mesmo que sua média na variável composta tenha sido considerada a maior.

Tabela 6 - Regressão Múltipla (método backward)

\begin{tabular}{|r|l|l|l|}
\hline Modelo & $\begin{array}{l}\text { Variáveis adicionadas } \\
\text { Identificação, }\end{array}$ & Variáveis retiradas & \multicolumn{1}{c|}{ Método utilizado } \\
\hline 1 & $\begin{array}{l}\text { Sensibilidade a preço, } \\
\text { Grupo de referência. }\end{array}$ & INSERIR \\
\hline 2 & Sensibilidade a preço & $\begin{array}{l}\text { Reverso (critério: } \\
\text { Probabilidade de F a ser } \\
\text { removido >= 100). }\end{array}$ \\
\hline
\end{tabular}

Fonte - Pesquisa de campo (2019). 
Na Tabela 7, é apresentado o resumo do modelo teórico. 0 valor do R 0,564, aponta a correlação das variáveis independentes com a variável dependente. As variáveis independentes explicam $31,8 \%$ da intenção de visitar, mensurado pelo $\mathrm{R}^{2}$. Porém, para ser considerada a relação entre as variáveis interdependentes com a dependente faz necessário considerar o valor do $\mathrm{R}^{2}$ ajustado, que nesse estudo representa 0,306 , tendo uma estimativa de erro padrão de 1,351, assim, o modelo teórico final (com Identificação e Grupos de Referência) explica 30,6\% das mudanças na variável dependente (Intenção de visitar eventos culturais em espaços públicos).

Tabela 7 - resumo do modelo

\begin{tabular}{|c|c|c|c|c|c|}
\hline Modelo & R & R2 & R2 ajustado & Estimativa do erro padrão & Durbin-Watson \\
\hline 1 & 0,573 & 0,328 & 0,310 & 1,347 & 1,677 \\
\hline 2 & 0,564 & 0,318 & 0,306 & 1,351 & \\
\hline \multicolumn{5}{|c|}{ R = Correlação entre as variáveis; $\mathrm{R}^{2}=$ percentual de quanto as variáveis independentes } \\
\multicolumn{3}{r|}{ explicam a VD; $\mathrm{R}^{2}$ ajustado = Correção feita no $\mathrm{R}^{2}$ quando se tem mais de uma VI. }
\end{tabular}

Fonte - Pesquisa de campo (2019).

A Tabela 8, apresenta detalhadamente os valores da ANOVA correspondente a 26,334, sendo a mesma entendida como uma forma de comparar a razão entre a variância a não variância (Field, 2009). Desse modo, foi possível identificar que o modelo teórico proposto neste trabalho, apresenta significância estatística. A partir das somas dos quadrados e do grau de liberdade é possível chegar à soma do quadrado médio, sendo calculado dividindo as somas do quadrado pelo grau de liberdade associado. Uma das partes mais importantes da tabela 5, é a razão F, seu cálculo utiliza a equação e a significância associada a esse valor $F$, sendo aqui o valor correspondente a 26,334, onde é significativo ao nível de $p<0,05$. Sendo assim, o Sig. na Tabela 5 corresponde a 0,000 , concluindo que o modelo de regressão resulta em uma boa intenção de visitação, ou seja, ao menos uma variável independente é decisiva para explicar o comportamento da variável dependente.

Tabela 8 - ANOVA

\begin{tabular}{|l|c|c|c|c|c|}
\hline Modelo & Soma dos Quadrados & Df & Quadrado médio & F & Sig. \\
\hline Regressão & 96,240 & 2 & 48,120 & 26,334 & 0,000 \\
\hline 2 & Residual & 206,483 & 113 & 1,827 & \\
\hline Total & 302,723 & 115 & & \\
\hline
\end{tabular}

A Tabela 9, apresenta os coeficientes de análise de regressão múltipla. Foi possível identificar a não influência da variável sensibilidade ao preço. Dessa forma, hipótese $\mathrm{H}_{1}$ que diz respeito a sensibilidade ao preço foi refutada $(\beta=-0,130 ; t=-1,323 ; p>0,05)$ não exercendo influência sobre a intenção de visitar eventos culturais em espaços públicos. Esse resultado levou ao entendimento de que, as pessoas que procuram eventos culturais em espaços públicos do Bairro do Recife, procuram por motivos que a princípio não são 
financeiros. Dessa forma, essa procura é impulsionada primeiramente por fatores que não envolvem a precificação. Portanto, compreende-se que as pessoas que frequentam os eventos no Bairro do Recife, não pelo fator preço ou porque elas estão fugindo de eventos pagos, mas, pelas características dos eventos do Bairro do Recife. A ideia apresentada no referencial teórico de que a sensibilidade ao preço influenciaria positivamente a intenção de visitar eventos culturais nos espaços públicos do Bairro do Recife não foi confirmada, não corroborando com a teoria de Lichtenstein et al. (1993), que diz respeito ao quanto as pessoas estão dispostas a procurarem por produtos mais econômicos e se realmente elas fazem um esforço para procurar por esses produtos. Para explicar o resultado da não confirmação da hipótese, uma possível teoria pode ser abordada. As novas tendências (Rasquilha, 2019), podem persuadir nas escolhas das principais aquisições dos consumidores. Nesse processo, não é levado primeiramente em consideração fatores de precificação, sendo considerados aqui, fatores emocionais e afetivos. Ao observarem as novas tendências que são acompanhadas pelos grupos de referência, os indivíduos podem ser influenciados, fazendo com que eles adquiriram os mesmos produtos ou frequentem os mesmos locais dos grupos de referência, independentemente de custos financeiros.

A Hipótese $\mathrm{H}_{2}$ que diz respeito aos grupos de referência (GDR) foi confirmada $(\beta=0,386 ; t=3,511 ; p<0,05)$, demonstrando sua influência sobre a intenção de visitar eventos culturais em espaços públicos do Bairro do Recife. 0 resultado levou ao entendimento que as pessoas escolhem participar de eventos culturais no Bairro do Recife, através das influências de grupos de pessoas, que podem ser do seu convívio diário, como familiares ou amigos do trabalho, como também de influenciadores digitais. Essa variável se torna relevante ao demonstrar a sua influência sobre a VD.

Dessa forma, foi possível concluir que a ideia apresentada no referencial teórico onde acreditava-se que, os grupos de referência influenciariam positivamente a intenção de visitar eventos culturais nos espaços públicos do Bairro do Recife, foi de encontro com a teoria de Park \& Lessig (1977), onde relatam que esses grupos de referência, possuem um papel de influenciadores e consideram fatores emocionais, culturais, sociais, pessoais e psicológicos. Assim, os indivíduos buscam por informações de produtos, lugares, marcas, através de pessoas que já fazem uso ou os escolhem a partir da utilização dos grupos de referência.

A hipótese $H 3$, que diz respeito à identificação (I), foi confirmada $(\beta=0,335$; $t=4,705 ; \mathrm{p}<0,05)$, e demonstrou a sua influência sobre a intenção de visitar eventos culturais em espaços públicos do Bairro do Recife. Logo, o resultado demonstrou que as pessoas que buscam ou frequentam os eventos culturais no Bairro do Recife, vão por se identificarem, seja, com o local, os monumentos históricos, as pessoas que circulam no bairro, ou pelo sentimento de pertença.

Pode-se concluir, que, essa variável exerce influência sobre a VD que é a intenção de visitar eventos culturais, e que a ideia apresentada no referencial teórico, onde a identificação influenciaria positivamente a intenção de visitar eventos culturais nos espaços públicos do Bairro do Recife, foi de encontro com a teoria de Algesheimer et al. (2005), que diz respeito ao quanto o indivíduo se identifica com a localidade. Explicando assim, tal resultado. 
Tabela 9 - Coeficientes

\begin{tabular}{|c|c|c|c|c|c|c|c|c|}
\hline & \multirow[t]{2}{*}{ Modelo } & \multicolumn{2}{|c|}{$\begin{array}{l}\text { Coeficientes não } \\
\text { padronizados }\end{array}$} & \multirow{2}{*}{$\begin{array}{c}\text { Coeficientes } \\
\text { padronizados } \\
\text { Beta }\end{array}$} & \multirow[t]{2}{*}{$\mathbf{t}$} & \multirow[t]{2}{*}{ Sig } & \multicolumn{2}{|c|}{$\begin{array}{c}\text { Intervalo de } \\
\text { confiança } 95,0 \% \\
\text { para B }\end{array}$} \\
\hline & & B & $\begin{array}{l}\text { Modelo } \\
\text { padrão }\end{array}$ & & & & $\begin{array}{l}\text { Limite } \\
\text { inferior }\end{array}$ & $\begin{array}{l}\text { Limite } \\
\text { superior }\end{array}$ \\
\hline & (Constante) & 2,562 & 0,549 & & 4,668 & 0,000 & 1,475 & 3,650 \\
\hline \multirow[t]{4}{*}{1} & $\begin{array}{l}\text { Sensibilidade } \\
\text { a preço }\end{array}$ & $-0,130$ & 0,098 & $-0,103$ & $-1,323$ & 0,189 & $-0,325$ & 0,065 \\
\hline & $\begin{array}{l}\text { Grupo de } \\
\text { referencia }\end{array}$ & 0,402 & 0,110 & 0,304 & 3,650 & 0,000 & 0,184 & 0,621 \\
\hline & Identificação & 0,326 & 0,071 & 0,380 & 4,571 & 0,000 & 0,185 & 0,467 \\
\hline & (Constante) & 2,169 & 0,463 & & 4,685 & 0,000 & 1,252 & 3,087 \\
\hline \multirow[t]{2}{*}{2} & $\begin{array}{l}\text { Grupo de } \\
\text { referencia }\end{array}$ & 0,386 & 0,110 & 0,291 & 3,511 & 0,001 & 0,168 & 0,603 \\
\hline & Identificação & 0,335 & 0,071 & 0,391 & 4,705 & 0,000 & 0,194 & 0,476 \\
\hline & $B=$ coeficient & ́o pa & $\begin{array}{l}\text { nizado; } \\
\text { si } \\
\text { Fonte - }\end{array}$ & $\begin{array}{l}\text { coeficiente p } \\
\text { icância ou P- } \\
\text { quisa de cam }\end{array}$ & oniza & ; $\mathrm{T}=\mathrm{t}$ & e t; sig = & ível de \\
\hline
\end{tabular}

Dessa forma, resumidamente, constatou-se a rejeição da $\mathrm{H}_{1}$ sensibilidade ao preço (SAP), onde se acreditava que esse construto influencia positivamente a intenção de visitar eventos culturais nos espaços públicos do Bairro do Recife. Porém, ele demonstrou ser inverso a intenção de visitar eventos culturais. $\mathrm{A} \mathrm{H}_{2}$, os grupos de referência (GDR) que acreditava conseguir influenciar positivamente na intenção de visitar eventos culturais nos espaços públicos do Bairro do Recife foi confirmada, indicando que o construto influencia positivamente na intenção de visitar. $\mathrm{A} \mathrm{H}_{3}$ a identificação, também foi confirmada, chegando à conclusão de que esse construto influencia positivamente na intenção de visitar eventos culturais nos espaços públicos do Bairro do Recife. Feito esse levantamento e as discussões cabíveis, a sessão a seguir será destinada à conclusão e as limitações deste trabalho.

\section{CONCLUSÕES}

Esta pesquisa teve por objetivo mensurar características comportamentais dos indivíduos que os levam a ter a intenção de visitar eventos culturais nos espaços públicos do Bairro do Recife. Dessa forma, se constatou em um primeiro momento, que a sensibilidade ao preço não exerce nesse estudo uma influência significativa sobre a intenção de visitar. Esse construto faz relação ao quanto as pessoas estão dispostas a procurarem preços mais baixos e se de fato elas procuram por preços mais acessíveis. Compreende-se, que as pessoas que frequentam os eventos culturais dos espaços públicos do Bairro do Recife, não possuem intenção de visitar eventos culturais em espaços públicos do Bairro do Recife, a princípio pelo financeiro, um dos principais motivos para essa intenção e visitação é pela influência dos grupos de referência, construto esse que teve sua aceitabilidade nesse estudo, demonstrando a sua influência na tomada de decisão dos indivíduos. Assim, os indivíduos acabam tomando decisões com relação 
à aceitação de outras pessoas. Portanto, esse construto influencia positivamente a intenção de visitar eventos culturais em espaços públicos do Bairro do Recife.

Essa mesma pessoa, também pode ser influenciada pela identificação com o local. Aspectos históricos, arquitetônicos, atraem e despertam no indivíduo uma sensação de pertença. Assim, como músicas, atratividades, os estilos de pessoas que circulam na localidade, entre tantos outros fatores que são determinantes para a tomada de decisão por identificação. Desse modo, o construto de identificação foi confirmado, demonstrando sua influência na tomada de decisão dos indivíduos. Logo, a principal contribuição desta pesquisa, é que a intenção de se visitar eventos culturais nos espaços públicos urbanos do Bairro do Recife se encontra atrelada a questões de influência por porte de grupos de pessoas e por questões de identificação, seja com o local, por possuir características únicas, ou pelas pessoas que circulam no seu entorno. O Bairro do Recife atualmente, passa por novos usos, onde empresas de tecnologia da informação e comunicação estão se instalando com maior frequência e tornando o bairro mais movimentado e utilizável, com parcerias público-privadas, essas empresas também podem assumir o seu papel como influenciadoras, tornando o ambiente do Bairro do Recife apropriado para esses eventos culturais. Dessa maneira, essas empresas tornam-se grupos de referência para os indivíduos, ao promover o ambiente do Bairro do Recife, ao mesmo tempo, elas tornam-se influenciadoras na identificação ao preservar com os órgãos públicos, os aspectos arquitetônicos e culturais do bairro. A amostra desta pesquisa, é não probabilística por conveniência do pesquisador, não é uma amostra que busca em sua finalidade generalizar as informações de que, todo o público que frequenta o Bairro do Recife, pensam dessa maneira, pelo contrário para esse recorte, esse público especifico, com essas características, ficou claro a sua posição com relação ao consumo de eventos culturais nos espaços públicos do Bairro do Recife.

Por fim, a relevância dessa pesquisa encontra-se na originalidade do estudo, por não possuir estudos na área de turismo que norteiam com tal enfoque essa temática. Sendo de grande importância para fonte de dados e estudos futuros que contemplem ainda mais essa pesquisa, de modo a proporcionar não só a área acadêmica, como também ao mercado, estatísticas que possam auxiliar a elaboração de estratégias mercadológicas e acadêmicas. Dessa forma, o estudo do ponto de vista acadêmico contribui para uma melhor análise das decisões de visitar eventos culturais em espaços públicos do Bairro do Recife, contribuindo para o entendimento desse comportamento. Tal estudo também contribui com informes que ajudam o mercado a organizar melhor suas estratégias de modo a atender as necessidades da população. Para pesquisas futuras, sugere-se que seja realizado uma pesquisa in loco, para verificar se os resultados sobrem algum tipo de alteração e assim possam ser confrontados.

\section{Limitações e sugestões para futuras pesquisas}

Uma limitação da pesquisa é que possui dados apenas referentes ao Bairro do Recife, limitando o alcance de generalização para outros contextos de eventos culturais. Além disso, o perfil da amostra com elevada renda individual mensal traz um contexto específico para o estudo, que serve para uma compreensão de 
uma parcela do público analisado, mas não permite fazer inferências para todos os públicos que frequentam as atividades do Bairro do Recife. A coleta de dados realizada através do processo de Snowball (bola de neve), pode ter direcionado uma amostra com o público com uma renda mais elevada. Como o estudo se limita ao Bairro do Recife, na região Pernambucana do nordeste brasileiro, sugere-se para estudos futuros realizar essa pesquisa in loco, a fim de verificar se esse comportamento prevalece ou sofre alguma alteração, para que possam ser confrontados os resultados e ampliar esse comportamento em outras regiões, para que dessa forma possa obter novos insights que contribuam com o estudo desse comportamento, comparando se há mudanças significativas de localidade para localidade. Também, sugere-se que sejam estudados os motivos pelos quais o construto de sensibilidade ao preço não foi confirmado e quais foram as suas limitações. Assim, como poderia ser realizada uma amostra mais ampla, in loco, com um público maior, para verificar se de fato o público tem esse perfil, ou esse perfil é uma característica da forma em que o dado foi coletado. Por fim, indica-se estudar a influência que as empresas de TIC, localizadas no Bairro do Recife, exercem sobre os eventos culturais públicos do bairro e quais estratégias mercadológicas estão por trás desse processo.

\section{AGRADECIMENTOS}

Os autores agradecem à Coordenação de Aperfeiçoamento Pessoal de Nível Superior (CAPES) pelo apoio da bolsa de estudos.

\section{REFERÊNCIAS}

Abreu, C. (2020). Análisis estructuralista de la teoría de los grupos de referência. Agora 39(1), 59-80. DOI: 10.15304/ag.39.1.5679.

Algesheimer, R., Dholakia, U. M., \& Herrmann, A. (2005). The social influence of brand community: Evidence from European car clubs. Journal of Marketing. 69(3), 19-34. Doi.org/10.1509/jmkg.69.3.19.66363

Bragaglia, A. P., \& Bastos, L. L. A. (2017). 0 valor de aprovação social no consumo e na publicidade: uma discussão à luz da ética. Revista Fragmentos de Cultura-Revista Interdisciplinar de Ciências Humanas. 27(2), 258-270. http://dx.doi.org/10.18224/ frag.v27i2.5061

Carmo, H., \& Ferreira, M. M. (2008). Metodologia da investigação: guia para autoaprendizagem. Repositório aberto universidade aberta.

Castells, M., \& Borjas, J. (1996). As cidades como autores políticos. Nova estudos CEBRAP. 45 (2), 152-166. Doi: http://dx.doi.org/10.9771/pcr.v11i1.26706

Costa, F., Costa, E. M., \& Silva, J. L. M. (2016). Elasticidade preço e renda da demanda por energia elétrica nas regiões brasileiras: uma abordagem através de painel dinâmico. Revista de Economia. 42 (3). http://dx.doi.org/10.5380/re.v42i3.36594

Dziuban, C. D., \& Shirkey, E. C. (1974). When is a correlation matrix appropriate for factor analysis? Some decision rules. Psychological Bulletin. 81 (6), 358. https://doi.org/ $10.1037 / \mathrm{h} 0036316$

Engel, J. F., Blackwell, R. E., \& Miniardi, P. W. (2005). Comportamento do consumidor. São Paulo: Pioneira Thomson Learning. 
Escalas, J. E., \& Bettman, J. R. (2003). You are what they eat: The influence of reference groups on consumers' connections to brands. Journal of Consumer Psychology. 13(3), 339-348. https://doi.org/10.1207/S15327663JCP1303_14.

Fávero, L. P., Belfiore, P., Silva, F. L., \& Chan, B. L. (2009). Análise de dados modelagem multivariada para tomada de decisões. Elsevier. ISBN: 978-85-352-3046-8.

Field, A. (2009). Descobrindo a estatística usando o SPSS-2. Bookman Editora.

Fonseca, M. J., \& Rossi, C. A. V. (1998). O estudo do envolvimento com o produto em comportamento do consumidor: fundamentos teóricos e proposição de uma escala para aplicação no Brasil. ENCONTRO ANUAL DA ANPAD-ENANPAD. 22. 15 fev. 2020, http://www.anpad.org.br/admin/pdf/enanpad1998-mkt-22.pdf

Getz, D. (2008). Event tourism: Definition, evolution, and research. Tourism Management. 29(3), 403-428. https://doi.org/10.1016/j.tourman.2007.07.017

Getz, D., \& Page, S. J. (2016). Progress and prospects for event tourism research. Tourism Management. 52, 593-631. https://doi.org/10.1016/j.tourman.2015.03.007

Hair, J. F., Black, W. C., Babin, B. J., Anderson, R. E., \& Tatham, R. L. (2009). Análise multivariada de dados. (2a ed.). São Paulo: Bookman Editora.

Han, S., Gupta, S., \& Lehmann, D. R. (2001). Consumer price sensitivity and price thresholds. Journal of Retailing. 77(4), 435-456. https://doi.org/10.1016/S0022-4359(01)00057-4

Henriques, E. B. (2003). A cidade, destino de turismo. Geografia: Revista da Faculdade de Letras da Universidade do Porto. 19. 0871-164X.

Horta, R. L., Schäfer, J. L., Coelho, L. R., Rodrigues, V. S., Oliveira, M. S., \& Teixeira, V. A. (2016). Condições associadas a prejuízo de desempenho em habilidades sociais em uma amostra de conveniência de usuários de crack. Caderno de saúde pública, 1-15.

Hutcheson, G. D., \& Sofroniou, N. (1999). The multivariate social scientist: Introductory statistics using generalized linear models. Manchester: Sage.

Inglis, B. G., \& Solomon, M. R. (1995). Ser e não ser: imagens de estilo de vida, grupos de referência e o agrupamento da América. Journal of Advertising. 24(1), 13-28. doi.org/ 10.1080/00913367.1995.10673465

Jansen-Verbeke, M. (1988). Leisure + shopping = tourism product mix. In: Ashworth, G. \& Goodall, B. Marketing tourism places. Londres: Routledge.

Leite, R. P., \& Peixoto, P. (2009). Políticas urbanas de patrimonização e contra revanchismo: o recife Antigo e a Zona Histórica da Cidade do Porto. Cadernos Metropole. 93-104. ISSN: 2236-9996

Lichtenstein, D. R., Ridgway, N. M., \& Netemeyer, R. G. (1993). Price perceptions and consumer shopping behavior: a field study. Journal of Marketing Research. 30(2), 234-245. https://doi.org/10.1177/002224379303000208

Liu, Y. D. (2014). Cultural events and cultural tourism development: Lessons from the European Capitals of Culture. European Planning Studies. 22(3), 498-514. https://doi.org/ 10.1080/09654313.2012.752442

Macedo, S. B., Gosling, M. D. S., \& Queiroz, D. C. (2017). Eventos culturais: meu dinheiro me diz aonde vou? Uma análise sob a ótica da Teoria da Identidade Social (SIT). Revista Hospitalidade. 14(1), 01-23. https://doi.org/10.21714/2179-9164.2017v14n1.755

Marciel, J. R. B., Francisco-Maffezzolli, E. C., \& Martins, E. (2018). Autenticidade de Lugar. Revista Turismo em Análise. 29(3), 413-427. http://dx.doi.org/10.11606/ issn.1984-4867.v29i3p413-427

Nascimento, A. M., Gouveia, V. V., Soares, A. K. S., Silva, J. B. S.; Grangeiro, A. S. (2017). Escala de necessidade de identificação: propriedades psicométricas em contexto brasileiro. 
Avaliação Psicológica: Interamerican Journal of Psychological Assessment. 16(3), 348-355. http://dx.doi.org/10.15689/ap.2017.1603.12883

Paiva, R. A. (2016). Turismo, produção e consumo do espaço. Turismo, arquitetura e cidade. In II Colóquio [inter]nacional sobre o comercio e cidade: uma relação de origem. (p. 33-54). São Paulo, SP.

Park, C. W., \& Lessig, V. P. (1977). Students and housewives: Differences in susceptibility to reference group influence.Journal of consumer Research. 4(2), 102-110. https://doi.org/ $10.1086 / 208685$

Pasquali, L. (1998). Análise fatorial: um manual teórico-prático. Brasília: Editora da UnB, no prelo.

Pearce, D. G. (2001). An integrative framework for urban tourism research. Annals of Tourism Research. 28(4), 926-946.

Porter, S. R., \& Whitcomb, M. E. (2005). Non-response in student surveys: The role of demographics, engagement and personality. Research in Higher Education. 46(2), 127-152. https://doi.org/10.1007/s11162-004-1597-2.

Putrevu, S., \& Lord, K. R. (1994). Comparative and noncomparative advertising. Attitudinal. 77-91. doi:10.1080/00913367.1994.10673443

Rasquilha, L. (2019). Coolhunting e pesquisa de tendências: observar, identificar e mapear as tendências e mentalidades emergentes do consumidor. Almedina.

Reis, E. A., Reis I. A. (2002). Análise Descritiva de Dados. Relatório Técnico do Departamento de Estatística da UFMG. 13 nov. 2019. www.est.ufmg.br

Sastre, P. T.N.; Serralvo, F.A.; Moras, A.C. (2010). A influência dos grupos de referência no processo decisório do consumidor: um estudo no segmento de veículos utilitários esportivos. Anais do Encontro de Marketing da ANPAD, 4, Florianópolis.

Sevcenko, N. (2002). 0 desafio das tecnologias à cultura democrática. Cidade e cultura: esfera pública e transformação urbana. 37-49.ISBN: 978-85-7448-065-5.

Souza, M. d., Guimarães, A. C., \& Araujo, C. d. (2013). Estresse no trabalho em professores universitários. Revista Brasileira de Ciências da Saúde, 1-8.

Trindade, A. C. D., \& Souza, I. C. M. (2018). A influência da marca no processo decisório de compra do consumidor no setor automobilístico. Opara. Revista de Ciências Contemporâneas Aplicadas. 8(1), 90-114.

Zapata Muñoz, C., Puerta Campiño, D. E., \& Torres Restrepo, E. M. (2018). Influencia de los grupos de referencia en el consumo se sustancias psicoactivas en seis adolescentes. (Doctoral dissertation). Corporación Universitaria Minuto de Dios.

Recebido em: $17 / 06 / 2020$

Aprovado em: 26/11/2020

\section{CONTRIBUIÇÕES}

Alessandra Souza Queiroz Melo: Definição do problema de pesquisa e objetivos, desenvolvimento da proposição teórica, realização da revisão bibliográfica e fundamentação teórica, escolha dos procedimentos metodológicos, coleta e análise de dados, elaboração de tabelas, gráficos e figuras, realização de cálculos e projeções, revisão crítica, redação e adequação do manuscrito às normas da RTA. 
Marconi Freitas da Costa: Definição do problema de pesquisa e objetivos, desenvolvimento da proposição teórica, realização da revisão bibliográfica e fundamentação teórica, escolha dos procedimentos metodológicos, coleta e análise de dados, elaboração de tabelas, gráficos e figuras, realização de cálculos e projeções, revisão crítica, redação e adequação do manuscrito às normas da RTA.

José Roberto Ferreira Guerra: Definição do problema de pesquisa e objetivos, desenvolvimento da proposição teórica e revisão crítica do manuscrito. 